\title{
Editorial
}

\section{The cell secretome in personalized and regenerative medicine}

In recent years, the secretome and exosomes derived from cells have been demonstrated to play an important role in regulating key processes within human tissues. Since 1998, the body of literature on these topics has grown exponentially. Over this period, the cells' secretome has been shown to be responsible for the regulation of cell survival, proliferation and differentiation, tumor growth and invasiveness, as well as a path for communication between the periphery and the central nervous system. Due to this increased attention to their role and impact on personalized and regenerative medicine strategies, we concluded that it would be timely to gather together articles from some of the leading groups in the exosome and secretome fields as a special issue of Biochimie. The main areas of focus include: 1) Proteomic, metabolomic, and lipidomic characterization of the secretome; 2 ) In vitro and in vivo studies on secretome mediated cell interactions; 3) Clinical Applications of secretome based therapies; 4) Modulation of the secretome. We have invited a panel of renowned scientists to join this special issue on "The Cell Secretome in Personalized and Regenerative Medicine". The editors would like to express their appreciation and gratitude to the panel of renowned scientists for their contributions to this special issue. We anticipate that the contents will prove to be timely and will contribute to future advances in this emerging field.
Finally, we would like to thank Malika Hassini and the production staff of Biochimie at Elsevier for their unwavering help in compiling and formatting this special issue.

António J. Salgado, Guest Editor* Life and Health Sciences Research Institute (ICVS), School of Medicine, University of Minho, Braga, Portugal

Jeffrey M. Gimble, Guest Editor LaCell LLC and Tulane University Center for Stem Cell Research \& Regenerative Medicine, New Orleans BioInnovation Center, 1441 Canal Street, New Orleans, LA, 70112, USA

Bruno M. Costa, Guest Editor Life and Health Sciences Research Institute (ICVS), School of Medicine, University of Minho, Braga, Portugal

* Corresponding author. E-mail address: asalgado@med.uminho.pt (A.J. Salgado).

Available online $\mathrm{xxx}$ 